\title{
Unsymmetrical Relaxation Paths of the Excited States in Cyanine Dyes Detected by Time-Resolved Fluorescence: Polymethinic and Polyenic Forms
}

\author{
Petro Lutsyk $^{1,2, *}$, Yuri Piryatinski ${ }^{1}$, Oleksiy Kachkovsky ${ }^{3}$ Anatoly Verbitsky ${ }^{1}$, Aleksey Rozhin ${ }^{2}$ \\ ${ }^{1}$ Institute of Physics, National Academy of Sciences of Ukraine, 46, prospekt Nauky, 03680 Kyiv, Ukraine. \\ ${ }^{2}$ Nanotechnology Research Group \& Aston Institute of Photonic Technologies, \\ School of Engineering \& Applied Science, Aston University, Aston Triangle, B4 7ET Birmingham, UK. \\ ${ }^{3}$ Institute of Bioorganic Chemistry and Petrochemistry, National Academy of Sciences of Ukraine, \\ 1, Murmanska str., 02660 Kyiv, Ukraine. \\ *Corresponding author; email: p.lutsyk@aston.ac.uk
}

\begin{abstract}
Novel applications of organic dyes and vast opportunities for their molecular tailoring keep focus of scientific community on the issues of symmetry breaking in the systems having different location of uncompensated charge, which has tremendous impact on photoluminescent properties of the dyes. In this paper, we provide distinctive experimental evidences of three relaxation paths (one symmetrical and two unsymmetrical) of excited states by analysis of lifetime and spectra of time-resolved fluorescence at low temperature with strong support of quantum-chemical modeling. Importantly, the studied cyanine dye (astraphloxin) in aqueous solution has two different unsymmetrical relaxation paths of excites states in the polymethinic and donoracceptor polyenic forms, where the last form strongly diminishes in less polar media. The experimental and computational results provide essential fundamental knowledge of molecular electronic relaxations substantially affected by matrix rigidity and polarity for design and photonic applications of elongated $\pi$ electronic systems.
\end{abstract}




\section{Introduction}

Well-known polymethine dyes, discovered in the middle of the XIX century, continue to hold an actual fascination of scientific community owing to their unique photonic and sensing properties. ${ }^{1-3}$ Particular prominence is gained by nitrogen containing polymethines, cyanine dyes. ${ }^{1-6}$ At present time, these dyes have found the renewal of interest for near-infrared (NIR) applications in bio-imaging, nonlinear optics, and design of advanced photonic materials. ${ }^{4-6}$ In the general form, ionic polymethine dyes have a positive (or negative) charge delocalized between two electron-donating (or electron-accepting) terminal groups (TGs) via an odd number of $\mathrm{sp}^{2}$-hybridising carbon atoms, as it is presented by formula $\mathbf{1}$ :

$\mathrm{TG}^{+/-}-(\mathrm{CH}=\mathrm{CH})_{\mathrm{i}}-\mathrm{CH}=\mathrm{TG} \quad \mathbf{1}$

where $i$ is a number of vinylene groups $(-\mathrm{CH}=\mathrm{CH}-),{ }^{1,4}$ The conjugated chain of carbon atoms between TGs forms a polymethine chain. Therein, the presence of an extensive $\pi$-electron system provides a comparatively low energy of the first electron transition between the frontier levels and hence the absorption/fluorescence in UV, visible, and NIR spectral ranges. Another feature of such ionic linear systems is a considerable redistribution of the electronic densities at the carbon atoms in the polymethine chain upon excitation that gives the large-scale transition dipole moment and hence a high intensity of the long wavelength spectral band..$^{1,4}$

Cyanine dyes became a prefect modelling system for photonic applications due to the existing knowledge on the systems. ${ }^{1,4,5}$ The lengthening of polymethine chain in such dyes (1) by increasing $i$ to $i+1$ regularly shifts the spectral band to longer wavelengths on approximately $100 \mathrm{~nm} .{ }^{4}$ The shift is complemented by broadening of the typical cyanine band with a sharp narrow maximum, whereas a new broad shoulder appears at the short wavelength side. ${ }^{7,8}$ Comprehensive spectral and quantum-chemical investigations have helped to establish that this phenomenon is associated with coexisting of two different forms of the dye having the long chain: with symmetrical and unsymmetrical charge distributions along the polymethine chain. ${ }^{4,7-13}$ Such mobility of the electronic charge distribution has been explained by a soliton conception. ${ }^{7-17}$ According to this conception, the charge in the collective $\pi$-electron systems produces a solitonic wave of partial charges, so that the charges at neighboring carbon atoms of the polymethine chain alternate considerably. Importantly, it was shown that the soliton is located in the center of an ionic linear unsubstituted conjugated system, although any TGs could cause the movement of solitonic wave to one of the chain ends when the crucial number of vinylene groups in the polymethine chain is achieved. ${ }^{7-17}$ In the case of unsymmetrical distribution of the electron density, the unsymmetrical form appears having an additional wide spectral band in the short wavelength region. ${ }^{4}$ 
The symmetry breaking appears not only in the ground state $\left(S_{0}\right)$, but also in the first excited state $\left(S_{1}\right)$. Detailed evidences for a 'two-state two-mode' model in polymethine cyanines were reported. ${ }^{18}$ It was shown that upon excitation to $S_{1}$ state, the dye molecule follows a path with two different vibrational modes populated sequentially. The first vibrational mode is fully symmetric and relaxes originally planar system out of the Franck-Condon (FC) region through skeletal stretching oscillations. However the second vibrational mode is asymmetric with predominant torsional motions around one of the central double bonds of the system. ${ }^{18}$ As a consequence of above two-mode vibrations, the system translates into $90^{\circ}$ twisted configuration, which cannot be observed by steady state fluorescence at room temperature. This way, the second relaxation path is connected with the symmetry breaking, similarly to the ground-state symmetrybroken polymethine dyes (1) containing crucial number of vinylene groups, $i$, in the chain. In our group earlier paper, ${ }^{19}$ the existence of one unsymmetrical relaxation path for symmetrical polymethine dyes was confirmed experimentally by the low temperature time-dependent fluorescence, where conformational transformations are impossible due to rigidization (freezing) of solvent matrix.

Theoretical and computational considerations of two relaxation paths (two modes) ${ }^{18}$ have been performed for symmetrical polymethine dyes with simplest TGs only (having one $\pi$-electron center and $\mathrm{N}\left(\mathrm{CH}_{3}\right)_{2}$ groups), whereas a lot of polymethine dyes contains complex heterocycles with extensive conjugated system. ${ }^{11,18-20}$ In addition, the importance of uncompensated charge location (solitonic wave) should be considered. As a rule, chemists draw molecular structures of ionic polymethine dyes by a resonance structure with localization of the total positive or negative charge at one of the TGs. For example, formula 1 represents the first TG that is treated as an acceptor group, whereas the second TG (with the same chemical constitution) referred as a donor group; and the polymethine chain is treated as a polyene. At the same time, the polymethine dye of unsymmetrical form could be also presented by the unsymmetrical resonance structure with location of charge at any carbon atom in the chain, for example, by formula $\mathbf{2}$ :

$\mathrm{TG}-{ }^{+-} \mathrm{CH}=\mathrm{CH}-(\mathrm{CH}=\mathrm{CH})_{\mathrm{i}-1}-\mathrm{CH}=\mathrm{TG}$

One can suppose that similar two unsymmetrical forms (with location of the total charge mainly on the TG or the chain) could be found in the excited state. There are no comprehensive studies evidencing the above model of various unsymmetrical relaxation paths of the excited states in polymethine molecules. In this paper, we present the results of systematic study of different relaxation paths in the excited state for cyanine dye, astraphloxin (AF), in water by time-resolved spectroscopy and by quantum-chemical modeling. Our results provide comprehensive evidences of different unsymmetrical relaxation paths of excites states in the polymethinic and polyenic forms due to unique combination of modelled systems and employed experimental methodology. The results evidencing various relaxation pathways with change of lifetimes, spectra, and molecular structures could be applied for design of novel photonic molecular systems. 


\section{Materials and methodology}

\subsection{Materials}

The cationic cyanine dye, astraphloxin (AF), was obtained from the Institute of Organic Chemistry (NASU, Ukraine); its chemical formula is pictured in Figure 1a. AF purity of $98 \%$ was determined by liquid chromatography and mass spectrometry (LC-MS). AF has been studied as a model representative of the trimethine polymethine dyes exhibiting highly intensive and selective band in the visible part of the absorption and fluorescence spectra. The advantage of AF is relatively simple molecular structure for quantum-chemical modelling, good solubility in water serving as a highly polar medium, and efficient transcis $^{21}$ isomerization. AF molecule dissociates in the water on the positively charged conjugated part with $\pi$ electron system and negatively charged counter-ion $\left(\mathrm{Cl}^{-}\right)$, and main spectral properties of the dye are determined by its conjugated part.

Sodium dodecyl sulfonate (SDS) and 4-cyano-4'-pentyl-biphenyl (5CB) were purchased from SigmaAldrich and Merck, respectively (purity of both ca. 99\%). SDS was admixed to aqueous solutions of AF $\left(10^{-6}\right.$ $\mathrm{M})$ at micellar concentration $\left(0.01 \mathrm{M}\right.$ being above critical micelle concentration (CMC) of $\left.8 \mathrm{mM}^{22,23}\right)$. Above CMC, SDS molecules form micelle around cyanine molecule in aqueous solution, ${ }^{24}$ with polar sulfonate groups tending to face outer surface and neutral part burying inside (see the scheme in Figure 1b). In addition, a SDS anion is a potential replacement of the AF counter-ion. The formation of micelles around AF was observed in our previous work. ${ }^{25}$ SDS, $5 \mathrm{CB}$ and chloroform were chosen for study of local microenvironment (viscosity, polarity, etc.) around AF affecting internal motions and electronic properties of the studied molecule.

(a)<smiles>CN(C(=CC=CC1=[N+](C)c2ccccc2C1(C)C)C(C)(C)C)c1ccccc1</smiles>

conjugated part

counter-ion

(b)

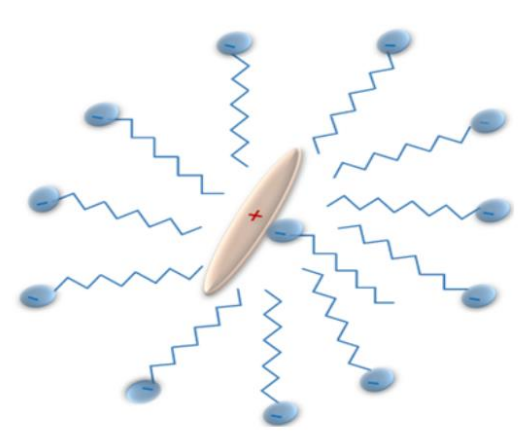

Figure 1. (a) Chemical formula of AF dye; (b) schematic illustration for the interaction of AF with SDS forming micelle around the dye.

\subsection{Quantum-chemical modeling}

The quantum-chemical calculations were performed by the Gaussian03 package. ${ }^{26}$ The counter ion $\left(\mathrm{Cl}^{-}\right)$has been omitted at the calculations, so far as we have studied solutions of AF at very low concentrations, where 
counter ion does not influence strongly on electron structure of the main chromophore. The optimized molecular geometry of the all-trans conjugated part of AF was obtained by the DFT//CAM-B3LYP/6-31(d,p) method. The characteristics of the electron transitions were calculated by both non-empirical TD DFT//CAMB3LYP/6-31(d,p) and semi-empirical ZINDO/S (overlap weighting factor, OWF, equal to 0.40; 25 lowest single excited configurations) methods. Of course, there is no perfect coinciding of the calculated and experimental data that is typical for polymethine dyes. ${ }^{27-29}$ However the modelling substantially supplements the experimental analysis of electron transitions and relaxation paths in the AF molecule.

\section{$\underline{2.3 \text { Experimental section }}$}

The absorption spectra were measured with a Lambda 1050 UV/VIS/NIR spectrometer (Perkin Elmer). The steady state fluorescence emission spectra were measured with USB2000+UV-VIS-ES spectrometer via $600 \mu \mathrm{m}$ optical fiber and with excitation of LLS-270 light-emitting diode (all Ocean Optics B.V.). The timeresolved fluorescence (time-resolved photoluminescence - TRPL) decays and time-resolved emission spectra (TRES) were measured with a LifeSpec II spectrofluorometer (Edinburgh Instruments Ltd) employing Time Correlated Single Photon Counting (TCSPC) with excitation by a picosecond pulse diode laser at wavelength $\lambda_{E X}=405 \mathrm{~nm}$ and pulse duration $40 \mathrm{ps}$ (EPL-405). TRES are spectral dependencies of fluorescence vs. various emission wavelengths $\left(\lambda_{E M}\right)$ or emission wavenumbers $\left(v_{E M}\right)$ at specific delay time, $t_{d}$. Our spectral studies for fluorescence at various wavelengths of excitation $\left(\lambda_{E X}\right)$ have shown that fluorescence spectra of $\mathrm{AF}$ at low concentration $\left(10^{-6} \mathrm{M}\right)$ are independent of $\lambda_{E X}$ in $\mathrm{UV}$ range. ${ }^{6,25}$

The lifetime data were obtained from exponential fitting of the fluorescence decays via FAST (Fluorescence Analysis Software Technology) and F900 software packages (both Edinburgh Instruments Ltd) taking into account Instrument Response Functions (IRF). Obtained experimental decays of fluorescence are fitted with multi-exponential model by formula $3^{30}$

$$
I(t)=\sum_{i=1}^{m} \alpha_{i} \exp \left(-t / \tau_{i}\right)
$$

where the sum of pre-exponential factors $\left(\alpha_{i}\right)$ is normalized to unity $\left(\Sigma \alpha_{i}=1\right)$ and $\tau_{i}$ are measured lifetimes of fluorescence. The $m$ is a number of lifetime components (or number of exponents in the fitting), and in our experiments $m$ was determined via sophisticated statistical analysis of lifetimes from the FAST program. The precision of fitted lifetimes for single or two-exponents was determined limiting the outcomes of fittings with $\chi^{2}$ in the range of $0.90-1.60$, whereas for three-exponential fitting we considered results with $\chi^{2}$ from 0.95 to 1.05. For fitted decays and TRES delay times, the count start (or maximum position of scattered laser pulse) was shifted to zero. According to the theory, ${ }^{30}$ lifetime of an excited molecular state in absence of nonradiative processes, $\tau_{0}$, is defined by formula 4

$$
1 / \tau_{0}=2.88 \times 10^{-9} n^{2}\left\langle v_{m}^{-3}\right\rangle^{-1} G \int \frac{\varepsilon(v) d v}{v}
$$

where $n$ is refractive index of the medium, $v_{m}$ is mean wavenumber of the absorption band in $\mathrm{cm}^{-1}, \varepsilon(v)$ is 
absorption coefficient, and $G$ is degree of degeneracy of the given state, equal to one for fluorescence. The presence of nonradiative processes is defined by the quantum yield of fluorescence, $Q$, and one obtains $\tau_{i}=$ $Q \times \tau_{0}$. Therefore, comprehensive analysis of time-resolved fluorescence has to be aligned with study of the quantum yield and absorption coefficient, which in turn depend on oscillator strength and environment of the molecules.

\section{Results and Discussion}

\subsection{Electron and geometrical structure of AF molecule}

The optimized spatial conformation of the conjugated part of all-trans AF conformer is presented in the Figure 2. One can see that all-trans AF molecule is practically planar, only 6 methyl groups $\left(\mathrm{CH}_{3}\right)$ are out-ofplane of the conjugated part of the conformer. Because of the polymethine chain is a main focus of our studies, we have analyzed closely the calculated parameters of carbon atoms neighboring and belonging to the chain (Figure 3, top inset).
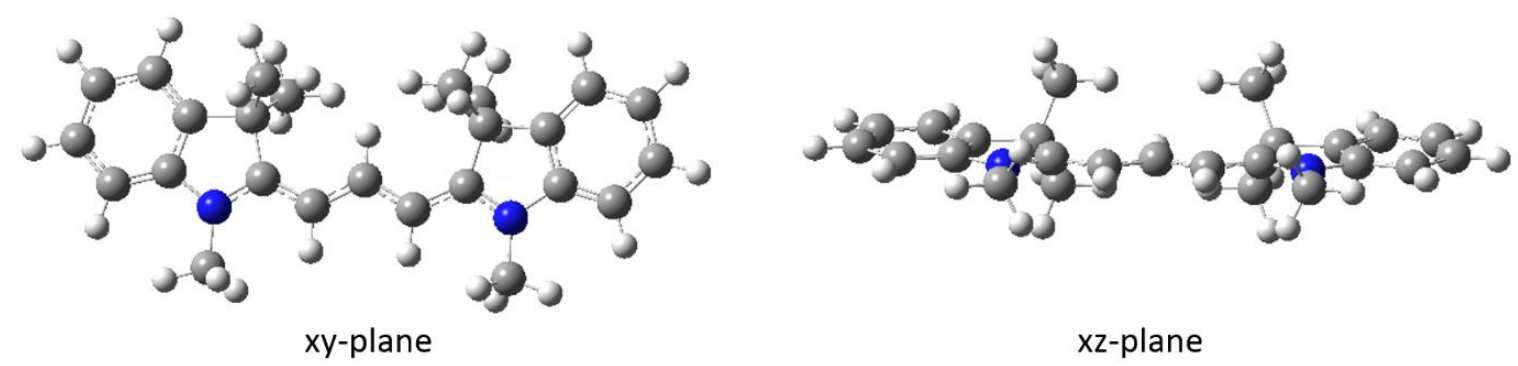

Figure 2. Spatial constitution of AF conjugated part in all-trans conformation.

All calculated parameters for AF (Figure 3), such as atomic charges, $q_{\mu}$, bond lengths, $l_{\mu v}$, and bond orders, $p_{\mu v}$, for both ground and excited states are characteristic for the polymethine dyes (see, for example, our previous results $\left.{ }^{4}\right)$. Therein, the optimized lengths of the carbon-carbon bonds $\left(l_{\mu v}\right)$ in $S_{0}$ of open polymethine chain are equalized (Figure $3 \mathrm{~b}$ ). Besides, the relatively high bond orders $\left(p_{\mu v}\right)$ are also practically equalized (Figure 3c). As a result, the barriers of conformational transformations in AF polymethine chain should be reasonably high, and possible cis-conformers or/and di-cis-conformers are practically absent (they are not spectrally observed, for example in NMR spectra ${ }^{4}$ ). In contrast to the equalized bonds, the atomic charges $\left(q_{\mu}\right)$ in the open polymethine chain are substantially alternated (Figure 3a) that has been confirmed experimentally ${ }^{4}$ by NMR spectroscopy. Importantly, the relatively large positive charges are located on the carbon atoms of terminal heterocycles adjacent to the nitrogen atoms, because of the substantial polarization 
of the $\mathrm{N}-\mathrm{C}$ bond. According to the calculations, the heterocyclic nitrogen atoms bear substantial negative charges (equal to -0.578 ).

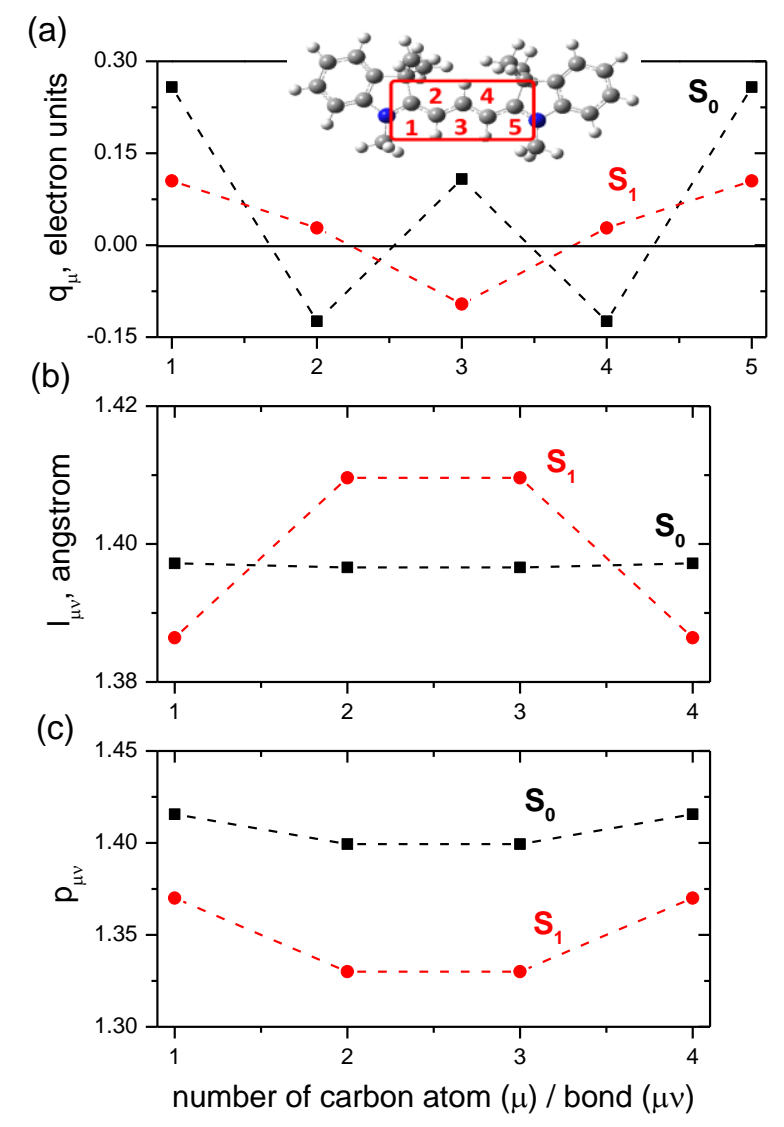

Figure 3. Atomic charges, $q_{\mu}$, (a), bond lengths, $l_{\mu v}$, (b), and bond orders, $p_{\mu v}$, (c) in polymethine chain (see inset at the top) of AF in ground ( $S_{0}$ - black) and Frank-Condon (FC) excited ( $S_{1}$ - red) state, where the numeration includes the carbon atoms of the TG connected to the polymethine chain. $l_{\mu \nu}$ in excited state (b) are obtained for relaxed excited state by optimization.

\subsection{Changes of electron structure upon excitation}

As a rule, the excitation of dye molecule by a quantum of light is accompanied by a substantial redistribution of the electronic occupancies at atoms and bonds in the dye chromophore. ${ }^{1,31}$ For AF, we will limit ourselves to the first electron transition, as far as it is the initial state for the fluorescence emission. The alternations of the atomic charges $\left(q_{\mu}\right)$ and bond orders $\left(p_{\mu v}\right)$ in the polymethine chain of the AF dye upon the first FrankCondon (FC) excited state $\left(S_{1}\right)$ are presented in the Figure $3 \mathrm{a}$ and $3 \mathrm{c}$, respectively. It should be mentioned that first electron transition occurs without change of equilibrium molecular geometry. ${ }^{30}$ Therefore, the bond 
lengths remain practically the same in both ground and FC excited state (Figure 3b). However, the excitation results in a small decrease of the bonds orders along the polymethine chain (Figure 3c). Essentially, the charges on carbon atoms undergo substantial alternations (Figure 3a), so the positive charge at the chain center (meso-position) reverses its sign and becomes negative. In turn, this results in change of a dipole moment of the molecule upon excitation influencing its solvation and other properties.

\subsection{Electron transitions and frontier molecular orbitals}

The shape of some molecular orbitals (MOs) closest to the energy gap and the excited configurations involved these MOs are pictured in the Figure 4, whereas characteristics of 6 lowest electron transitions are summarized in Table 1. Both frontier MOs (HOMO and LUMO) are substantially localized on the carbon atoms of the polymethine chain, and, in addition, some frontier and nearest MOs are delocalized along both polymethine chain and/or indolenine TGs (Figure 4). The symmetries of MOs alternate by-turn. Although, the HOMO-2 and HOMO-3 are located mainly on benzene rings of the TGs and might be treated as local MOs; they are practically degenerated. The results show that the first 5 electron transitions $\left(S_{0} \rightarrow S_{1}, \ldots\right.$, $S_{0} \rightarrow S_{5}$ ) correspond to electron transfers from 5 highest occupied orbitals to the same LUMO, and only sixth transition, $S_{0} \rightarrow S_{6}$, involves the HOMO and next vacant unoccupied MO (LUMO+1).

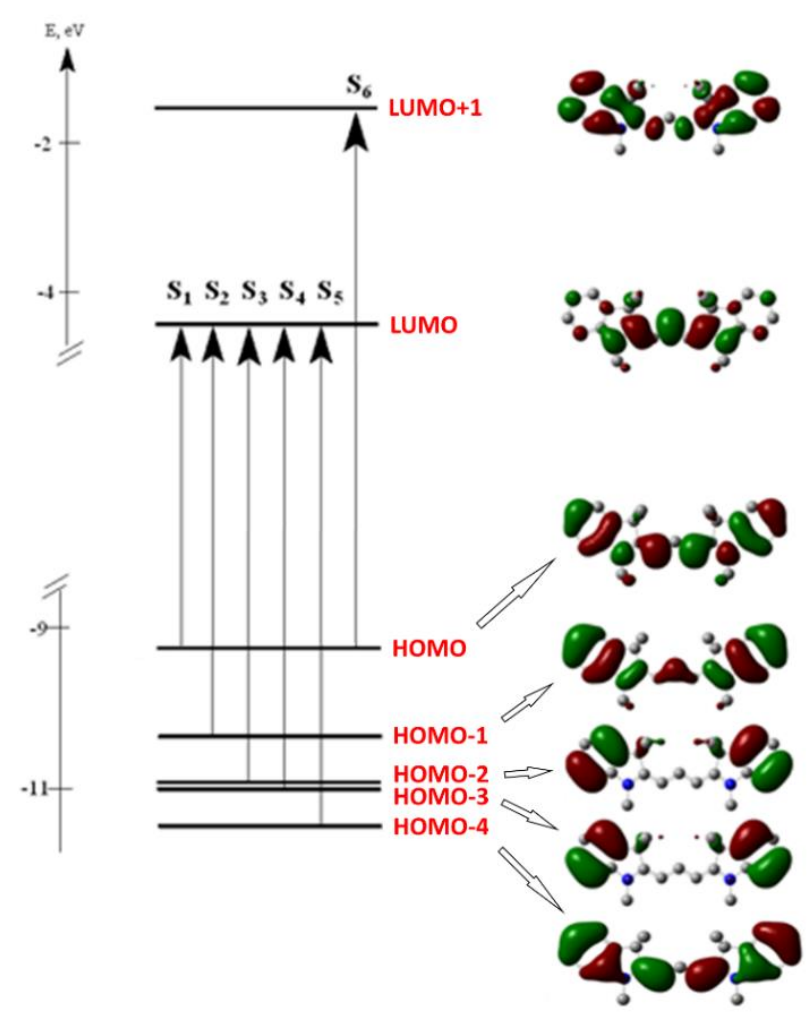

Figure 4. Frontier and nearest MOs as well as electron transitions between them in AF 
Table 1. Calculated characteristics of the six lowest transitions in AF (TD DFT and ZINDO/S methods)

\begin{tabular}{|c|c|c|c|c|c|c|}
\hline \multirow{2}{*}{$\begin{array}{l}\text { Transition } \\
\text { (Figure 4) }\end{array}$} & \multirow[b]{2}{*}{ Symmetry } & \multicolumn{2}{|c|}{ TD DFT } & \multicolumn{2}{|c|}{ ZINDO/S* } & \multirow{2}{*}{$\begin{array}{c}\text { Main configuration } \\
\text { with CI coefficient } \\
\text { (in parentheses) }\end{array}$} \\
\hline & & $\begin{array}{c}\text { wavenumber, } \\
\mathrm{cm}^{-1}(\mathrm{~nm})\end{array}$ & $\begin{array}{l}\text { oscillator } \\
\text { strength }\end{array}$ & $\begin{array}{c}\text { wavenumber, } \\
\mathrm{cm}^{-1}(\mathrm{~nm})\end{array}$ & $\begin{array}{l}\text { oscillator } \\
\text { strength }\end{array}$ & \\
\hline$S_{0} \rightarrow S_{1}$ & $A_{1} \rightarrow B_{1}$ & $23923(418)$ & 1.401 & $18248(548)$ & 0.969 & HOMO $\rightarrow$ LUMO (0.99) \\
\hline$S_{0} \rightarrow S_{2}$ & $A_{1} \rightarrow B_{1}$ & $34722(288)$ & 0.009 & $23256(430)$ & 0.042 & HOMO-1 $\rightarrow$ LUMO (0.97) \\
\hline$S_{0} \rightarrow S_{3}$ & $A_{1} \rightarrow A_{1}$ & $37879(264)$ & 0.029 & - & - & HOMO-2 $\rightarrow$ LUMO $(0.91)$ \\
\hline$S_{0} \rightarrow S_{4}$ & $A_{1} \rightarrow B_{1}$ & $38168(262)$ & 0.031 & - & - & HOMO-3 $\rightarrow$ LUMO (0.90) \\
\hline$S_{0} \rightarrow S_{5}$ & $A_{1} \rightarrow A_{1}$ & $41152(243)$ & 0.072 & - & - & HOMO-4 $\rightarrow$ LUMO (0.93) \\
\hline$S_{0} \rightarrow S_{6}$ & $A_{1} \rightarrow B_{1}$ & $43103(232)$ & 0.046 & - & - & $\mathrm{HOMO} \rightarrow \mathrm{LUMO}+1(0.72)$ \\
\hline
\end{tabular}

Similarly to other cationic polymethine dyes, the first transition, $S_{0} \rightarrow S_{1}$, in the AF has a large oscillator strength that is in a good agreement with the high intensity of the long wavelength band in the absorption spectra of such dyes. ${ }^{1,431}$ The next transitions have very low oscillator strengths and hence practically do not appear in the absorption spectra.

\subsection{Absorption and steady state fluorescence spectra}

The absorption spectrum of the aqueous solution of molecular AF (trans-isomer) at room temperature (Figure 5) has an intensive long wavelength band with the maximum at $18520 \mathrm{~cm}^{-1}(540 \mathrm{~nm})$. The spectral band in the absorption spectrum corresponds to the first electronic transition involving the HOMO $\rightarrow$ LUMO of opposite symmetries $\left(S_{0} \rightarrow S_{1}\right.$, Table 1$)$, which is antisymmetric $\left(A_{1} \rightarrow B_{1}\right)$ and hence is polarized along the polymethine chain. ${ }^{31}$ As we have seen, the oscillator strength of the long wavelength transition is the highest in comparison with higher-order excited electronic transitions in the spectrum (Table 1). At the short wavelength side, there is a weak shoulder at $19650 \mathrm{~cm}^{-1}(509 \mathrm{~nm})$ that is associated with the vibrational transitions. The broken-symmetry states contribute in the range of the vibrational transitions for molecules with long polymethine chain emitting in NIR range, ${ }^{4,12,13}$ which is not the case of AF. The study of absorption spectra of the $\mathrm{AF}$ in water solution at various concentrations has shown that the formation of dimers and $\mathrm{H}-$ aggregates is possible only at concentrations ca. $10^{-2} \mathrm{~mol} / \mathrm{L} \cdot{ }^{6,32} \mathrm{We}$ suppose that the spatial hindrances created by bulk methyl $\left(\mathrm{CH}_{3}\right)$ groups and/or out-of-plane molecular structure (see Figure 2) with possibility of slight rotational fluctuations of its TGs limit the minimum distance between AF molecules and hence prevent the aggregation at low concentrations. One notes that the spectral shoulder with the peak at $19650 \mathrm{~cm}^{-1}(509 \mathrm{~nm})$ [shifted hypsochromically on $1130 \mathrm{~cm}^{-1}$ relatively to the maximum at $18520 \mathrm{~cm}^{-1}(540 \mathrm{~nm})$ ] does not 
correspond to the second electronic transition, $S_{0} \rightarrow S_{2}$. Hence the shoulder is associated with the first vibrational $0 \rightarrow 1^{`}$ transition that is a typical for most of cyanine dyes. ${ }^{4}$

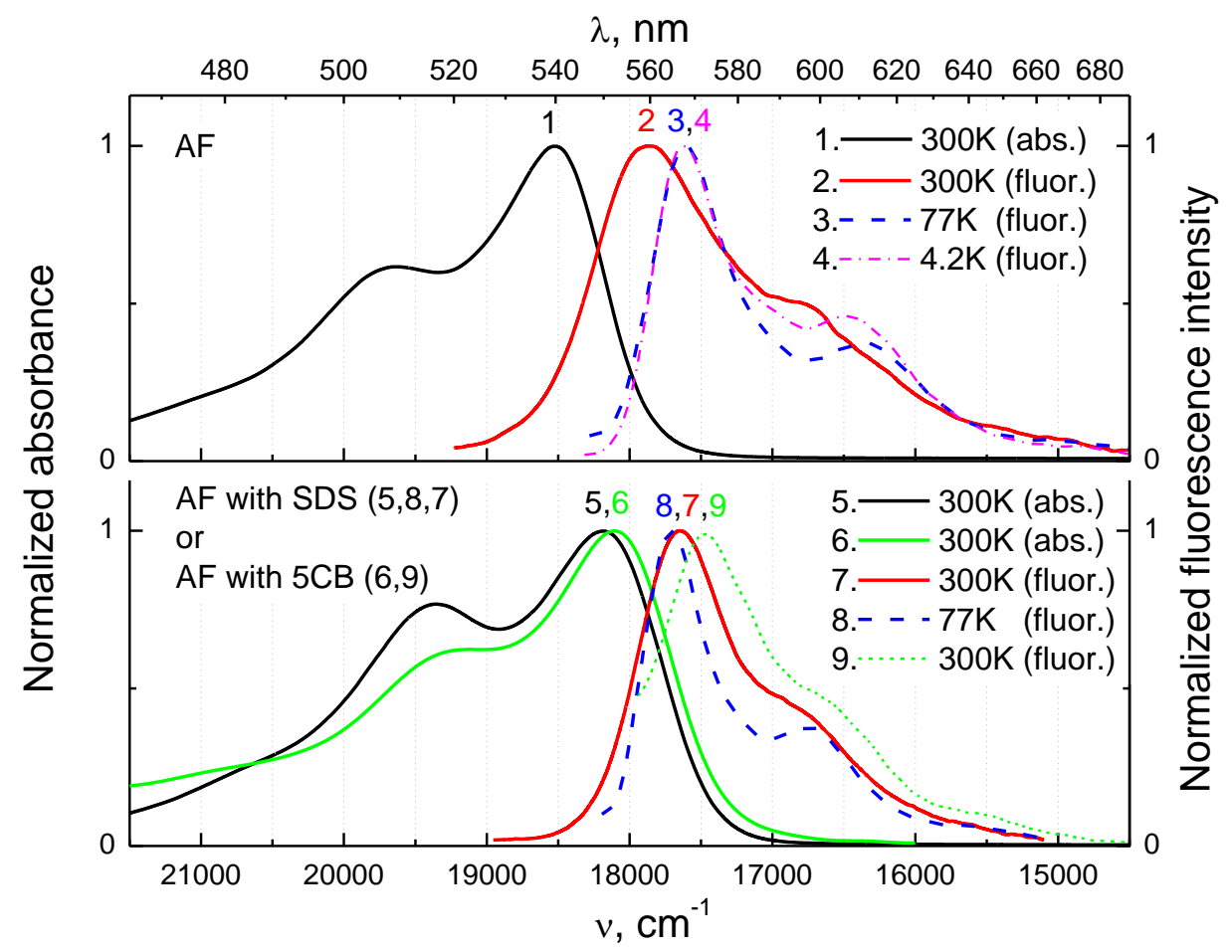

Figure 5. Molecular absorption spectra for aqueous solutions of neat AF (1), mixtures of AF with SDS (5) and solutions of AF in 5CB (6). Steady state fluorescence spectra $\left(\lambda_{E X}=270 \mathrm{~nm}\right)$ for aqueous solutions of neat AF (2-4), mixtures of AF with SDS $(7,8)$ and solutions of AF in 5CB (9) at $300 \mathrm{~K}(2,7,9), 77 \mathrm{~K}(3,8)$, and $4.2 \mathrm{~K}(4)$.

Comparison of the experimental absorption spectrum in the Figure 5 with calculated data in the Table 1 reveals that there is appreciable divergence between the calculated and experimental positions of the long wavelength band maximum. This is a typical drawback of the TD DFT method for the calculations of polymethine dyes. ${ }^{27-29}$ In fact, the calculations by semi-empirical ZINDO/S gives the absorption maximum at $481 \mathrm{~nm}$ in standard version and at $548 \mathrm{~nm}$ upon applied parameter of OWF $=0.4$ and 25 single excited configurations (Table 1). Thus, the semi-empirical calculations show precise nature of the electron transitions (including frontier MOs distributions, energy, and oscillator strength).

The steady state fluorescence maximum for aqueous solution of $\mathrm{AF}$ at room temperature is at $17860 \mathrm{~cm}^{-1}(560 \mathrm{~nm})$ (Figure 5, curve 2$)$ with relatively low quantum yield $(\approx 0.03)$. The low quantum yield of fluorescence is related to efficient trans-cis transformations of AF upon unsymmetrical path of 
radiationless relaxation. ${ }^{18,24}$ At the low temperature (77 and 4.2K), the fluorescence band maximum of $\mathrm{AF}$ is redshifted to $17610 \mathrm{~cm}^{-1}(568 \mathrm{~nm})$, as well as the spectral band becomes more narrow (Figure 5, curves 3,4). Importantly, that the quantum yield at the low temperatures is much higher (approximately 1). At low temperatures, the AF molecule in the frozen solution become rigid, hence structural difficulties arise for trans-cis isomerization responsible on radiationless relaxation. Such quantum yield enhancement due to the rigidization effect was also observed for other polymethine dyes. ${ }^{3,24,33}$

To study the effect of environment on relaxation paths for excited states, we have considered AF enclosed by SDS micelle (see Figure 1b) as the system of the same dye molecule having a local non-polar medium around. The absorption and steady state fluorescence spectra for the mixture of AF with SDS at micellar concentration in water are presented in Figure 5 (curves 5,7,8). The absorption maximum for the mixture at $18180 \mathrm{~cm}^{-1}(550 \mathrm{~nm})$ is $340 \mathrm{~cm}^{-1}$ redshifted due to the presence of SDS around AF in water (Figure 5, curves 1,5). The fluorescence maximum of the mixture at $300 \mathrm{~K}$ is $320 \mathrm{~cm}^{-1}$ redshifted to 17540 $\mathrm{cm}^{-1}(570 \mathrm{~nm})$ comparing to the maximum of neat AF (Figure 5, curves 2,7). We relate such redshifts to local change of relative electrical permittivity around $\mathrm{AF}$ and potential conformational constraints due to the presence of micelle. However complementary measurements of AF in non-polar chloroform solution gives even stronger redshift $\left(500 \mathrm{~cm}^{-1}\right)$ of absorption maximum up to $18020 \mathrm{~cm}^{-1}(555 \mathrm{~nm})$ with the fluorescence peak position at $17540 \mathrm{~cm}^{-1}(570 \mathrm{~nm})$ same as AF with SDS. Thus the local change of polarity has dominant contribution to the redshifts of the spectra in the SDS presence. In addition, the fluorescence intensity increases in 3 times upon formation of SDS micelles around AF and even slightly more for the AF in nonpolar chloroform. However at low temperatures, the fluorescence maxima (and spectral shapes) of neat AF and mixture of AF with SDS are practically the same peaking at $17610 \mathrm{~cm}^{-1}(568 \mathrm{~nm}$ ) (Figure 5, curves $3,4,8)$. Spectrally, rigidization of aqueous medium at low temperature makes systems of both AF enclosed by polar water and non-polar shell of SDS micelle very similar. Here, we revealed that there are same relaxation pathways in the frozen matrixes regardless of their polarity. Nevertheless, the difference of these two systems at low temperatures has been observed in time-resolved fluorescence and will be discussed below.

To prove the effect of rigid environment regardless its temperature, we have introduced AF molecules into highly viscous matrix of $5 \mathrm{CB}$ liquid crystal. $5 \mathrm{CB}$ at room temperature is more rigid and less polar than water medium. ${ }^{34}$ The quantum yield of $\mathrm{AF}$ in $5 \mathrm{CB}$ is close to 1 . The absorption and fluorescence maximum for neat $\mathrm{AF}$ in isotropic $5 \mathrm{CB}$ matrix at $18100 \mathrm{~cm}^{-1}(552 \mathrm{~nm})$ and $17320 \mathrm{~cm}^{-1}(577 \mathrm{~nm})$ at $300 \mathrm{~K}$, respectively (Figure 5, curves 6,9). This results in $420 \mathrm{~cm}^{-1}$ absorption redshift and $540 \mathrm{~cm}^{-1}$ fluorescence redshift in comparison to the spectra of neat $\mathrm{AF}$ in water. We relate the redshifts to low polarity and conformational constraints of 5CB. Comparison of absorption spectra for all studied systems (Figure 5) shows that except the redshifts the $19340 \mathrm{~cm}^{-1}(517 \mathrm{~nm})$ shoulder is considerably elevated for AF with SDS (Figure 5, curve 5) evidencing a specific interaction between the dye and SDS, potentially it could be a case displayed in Figure $1 \mathrm{~b}$ or a very weak $\mathrm{H}$-aggregation of AF by SDS molecule. Thus, the results evidence that in the highly 
viscous medium of $5 \mathrm{CB}$ as well as low temperature rotational trans-cis isomerization is highly obstructed preventing radiationless pathways for excited state relaxation.

\subsection{Time-resolved fluorescence decays}

The knowledge of time-resolved fluorescence features is very advantageous for all studied systems as it helps to analyze the lifetimes and to estimate spectral contributions from each relaxation pathway with determined lifetime. Therefore, to examine comprehensively the change of fluorescence spectra at matrix rigidification and polarity alternation the fluorescence decay kinetics were analyzed (Figure 6). All kinetics with only exception for AF at $77 \mathrm{~K}$ in water (Figure 6c) were well fitted with one or two exponents. The FAST statistical analysis of decays for AF at $77 \mathrm{~K}$ in water helped to reveal that these kinetics are best fitted with three exponential components. The lifetimes determined from the Figure 6 by exponential fitting decays as well as FAST intervals of lifetime distributions are summarized in the Table 2.

At room temperature, AF molecule is characterized not only by the low quantum yield $(\approx 0.03)$, but also by short lifetime - approximately 40 ps (curve 1 in Figure 6a). Thus, non-rigid form of aqueous matrix allowing trans-cis isomerization of AF not only leads to a low quantum yield of fluorescence, but also to a very short lifetimes. The introduction of the SDS micelles into AF molecular solution (with quantum yield rise up to approx. 0.09) increases lifetime to $120 \mathrm{ps}$ (curve 2 in Figure 6a), whereas the lifetime of $\mathrm{AF}$ in 5CB (with quantum yield reaching 1) increases considerably - up to $2.5 \mathrm{~ns}$ (Figure 6b). Thus, we can clearly see that at the matrix rigidification results in increase of lifetime and quantum yield of fluorescence. Fitting of $\mathrm{AF}$ in non-polar chloroform with lifetime of $270 \mathrm{ps,} \mathrm{we} \mathrm{have} \mathrm{evidenced} \mathrm{an} \mathrm{increase} \mathrm{of} \mathrm{lifetime} \mathrm{due} \mathrm{to} \mathrm{lowering}$ polarity of environment, which could be a result of the excited state stabilization by non-polar medium. ${ }^{35}$

However the most interesting result was observed for fluorescence decays at low temperatures. The kinetic fluorescence decay of neat $\mathrm{AF}$ (Figure 6c) is well fitted by 3 -exponential function with $\tau_{1}, \tau_{2}, \tau_{3}$, whereas kinetics for the mixture of AF with SDS are fitted by 2-exponential function with $\tau_{1}, \tau_{2}$. The lifetime analysis (Table 2) shows that the systems of AF with SDS and AF in 5CB have no long time component $\left(\tau_{3}\right)$ in the range of 3.5-10 ns. In addition, the pre-exponential factor for the slow $\left(\tau_{2}\right)$ component of AF in SDS micelles is $\alpha_{2}=0.86$, which is even higher than the sum of the $\alpha_{2}$ and $\alpha_{3}$ for neat AF (in the range of 0.710.81 ). Matrix rigidity at $77 \mathrm{~K}$ for both systems is practically the same, and the obvious difference is local polarity of medium around. Therefore, non-polar medium of SDS results in two radiative pathways with considerable predominance of the slow $\left(\tau_{2}\right)$ component. Essentially, in highly polar medium of water, at least three radiative pathways have been revealed for excited state relaxation in AF molecule: fast $-\tau_{1}$ ranging from tens to hundreds ps, intermediate $-\tau_{2}$ in the range of 1-3 ns, and slow $-\tau_{3}$ exceeding $3 \mathrm{~ns}$. The last $\left(\tau_{3}\right)$ radiative pathway is definitely induced by the polar environment of AF molecule. 
(a)
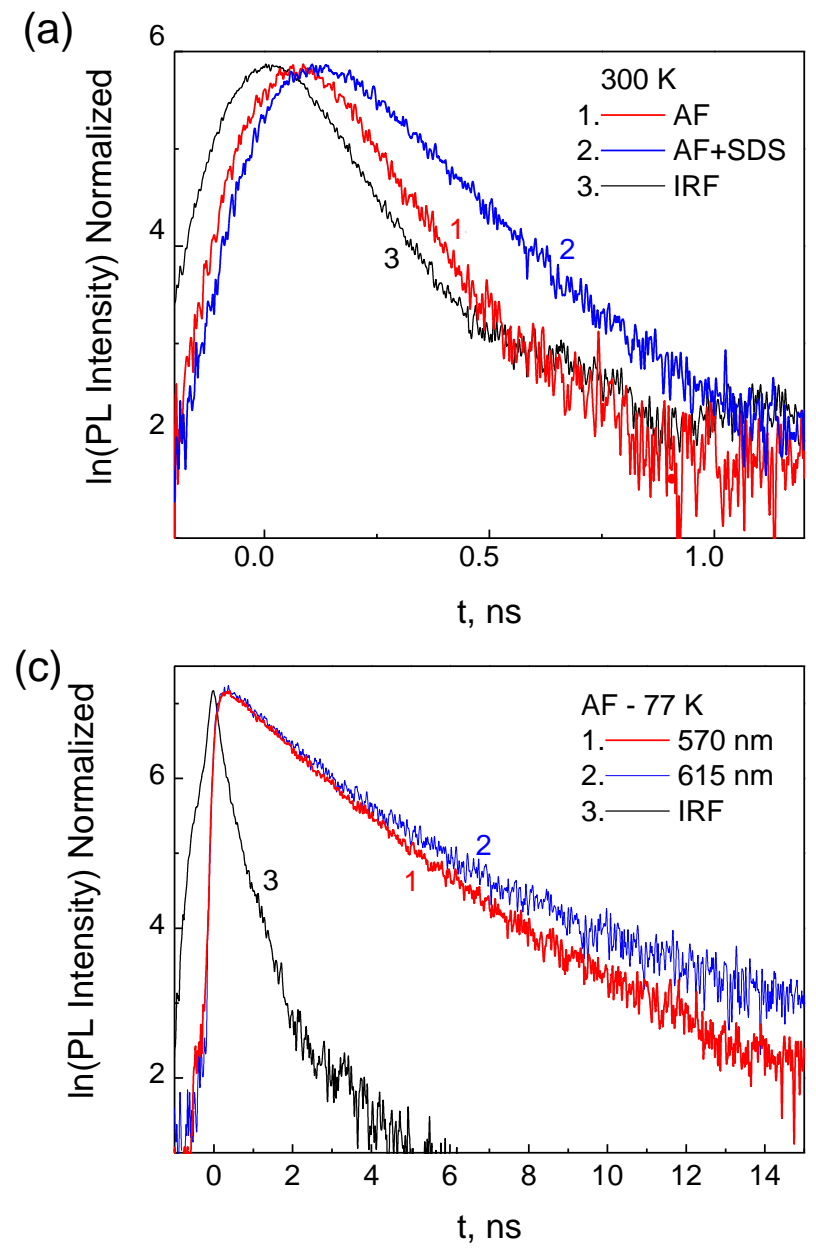

(b)

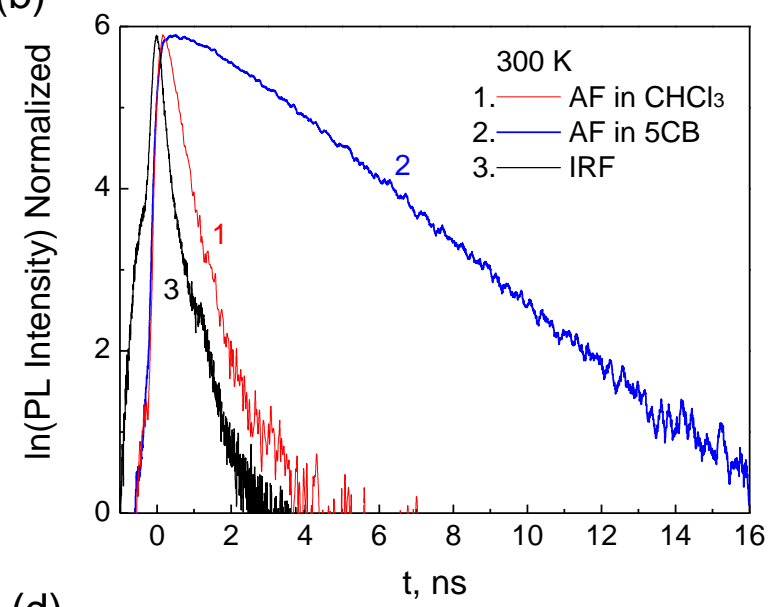

(d)

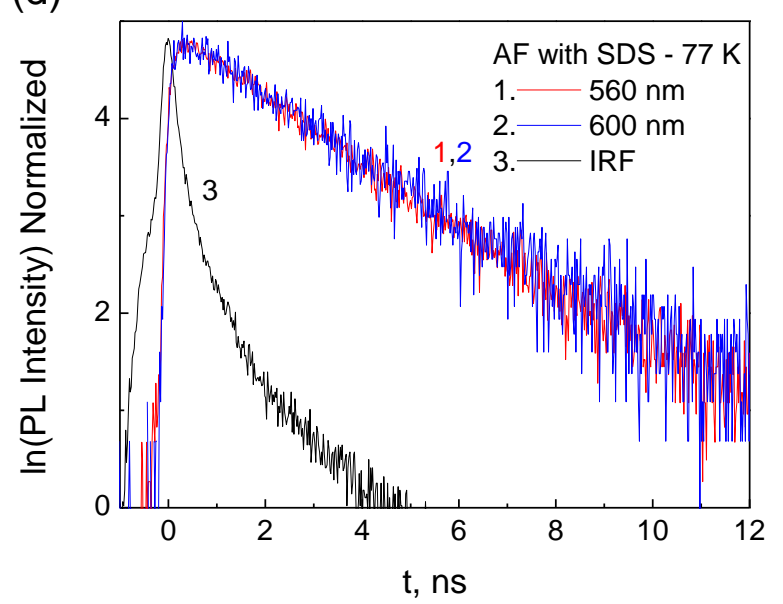

Figure 6. Time-resolved fluorescence decays $\left(\lambda_{E X}=405 \mathrm{~nm}\right)$ for aqueous solutions of AF $\left(\mathrm{a}-\right.$ curve $1\left(\lambda_{E M}=560 \mathrm{~nm}\right) ; \mathrm{c}-$ curves $1\left(\lambda_{E M}=570 \mathrm{~nm}\right)$ and $\left.2\left(\lambda_{E M}=615 \mathrm{~nm}\right)\right)$, mixtures of AF with SDS (a - curve $2\left(\lambda_{E M}=572 \mathrm{~nm}\right) ; \mathrm{d}$ - curves $1\left(\lambda_{E M}=560 \mathrm{~nm}\right)$ and $2\left(\lambda_{E M}=600 \mathrm{~nm}\right)$ ), chloroform solutions of AF $\left(\mathrm{b}-\right.$ curve $\left.1\left(\lambda_{E M}=590 \mathrm{~nm}\right)\right)$, AF in $5 \mathrm{CB}\left(\mathrm{b}-\right.$ curve $\left.2\left(\lambda_{E M}=578 \mathrm{~nm}\right)\right)$, and Instrument Response Functions (IRF) - (a-d - curve 3) at $300 \mathrm{~K}(\mathrm{a}, \mathrm{b})$ and $77 \mathrm{~K}(\mathrm{c}, \mathrm{d})$. 
Table 2. Lifetimes of fluorescence for AF in water, chloroform, 5CB, and aqueous mixtures of AF with SDS obtained from exponential fitting of TCSPC decays taking into account Instrument Response Function (IRF) and considering intervals of lifetime distributions from FAST software analysis.

\begin{tabular}{|c|c|c|c|c|c|c|c|c|}
\hline & \multirow{2}{*}{$\begin{array}{l}v_{E M}, \mathrm{~cm}^{-1} \\
\left(\lambda_{E M}, \mathrm{~nm}\right)\end{array}$} & \multirow{2}{*}{$\begin{array}{c}\tau_{1}, \mathrm{ps} \\
\left(\alpha_{1}\right)\end{array}$} & \multirow{2}{*}{$\begin{array}{c}\tau_{2}, \mathrm{~ns} \\
\left(\alpha_{2}\right)\end{array}$} & \multirow{2}{*}{$\begin{array}{c}\tau_{3}, \mathrm{~ns} \\
\left(\alpha_{3}\right)\end{array}$} & \multirow{2}{*}{$\chi^{2}$} & \multicolumn{3}{|c|}{ FAST distribution } \\
\hline & & & & & & $\tau_{1}, \mathrm{ps}$ & $\tau_{2}, \mathrm{~ns}$ & $\tau_{3}, \mathrm{~ns}$ \\
\hline $\begin{array}{l}\mathrm{AF}, \\
300 \mathrm{~K}\end{array}$ & $\begin{array}{l}17857 \\
(560)\end{array}$ & 40 & - & - & $\begin{array}{c}1.12 \\
\text { (Fig. 6a) }\end{array}$ & $30-50$ & - & - \\
\hline $\begin{array}{l}\text { AF with } \\
\text { SDS, } 300 \mathrm{~K}\end{array}$ & $\begin{array}{l}17482 \\
(572)\end{array}$ & 120 & - & - & $\begin{array}{c}1.22 \\
\text { (Fig. 6a) }\end{array}$ & $100-200$ & - & - \\
\hline $\begin{array}{l}\text { AF in } \mathrm{CHCl}_{3} \text {, } \\
300 \mathrm{~K}\end{array}$ & $\begin{array}{l}16950 \\
(590)\end{array}$ & 270 & - & - & $\begin{array}{c}1.16 \\
\text { (Fig. 6b) }\end{array}$ & $200-350$ & - & - \\
\hline $\begin{array}{l}\mathrm{AF} \text { in } 5 \mathrm{CB}, \\
300 \mathrm{~K}\end{array}$ & $\begin{array}{l}17301 \\
(578) \\
\end{array}$ & - & 2.50 & - & $\begin{array}{c}1.54 \\
\text { (Fig. 6b) }\end{array}$ & - & $2.0-3.0$ & - \\
\hline \multirow{2}{*}{$\begin{array}{l}\text { AF, } \\
77 \mathrm{~K}\end{array}$} & $\begin{array}{l}17543 \\
(570)\end{array}$ & $\begin{array}{c}160 \\
(0.29)\end{array}$ & $\begin{array}{c}1.68 \\
(0.36)\end{array}$ & $\begin{array}{c}\mathbf{3 . 5 3} \\
(\mathbf{0 . 3 5})\end{array}$ & $\begin{array}{c}1.04 \\
\text { (Fig. 6c) }\end{array}$ & \multirow{2}{*}{$150-300$} & \multirow{2}{*}{$1.5-2.6$} & \multirow{2}{*}{$3.5-10$} \\
\hline & $\begin{array}{l}16260 \\
(615)\end{array}$ & $\begin{array}{c}\mathbf{2 1 0} \\
(0.19)\end{array}$ & $\begin{array}{c}\mathbf{1 . 6 0} \\
(0.45)\end{array}$ & $\begin{array}{c}\mathbf{4 . 0 5} \\
(0.36)\end{array}$ & $\begin{array}{c}0.95 \\
\text { (Fig. 6c) }\end{array}$ & & & \\
\hline $\begin{array}{l}\text { AF with } \\
\text { SDS, } 77 \mathrm{~K}\end{array}$ & $\begin{array}{l}16667 \\
(600)\end{array}$ & $\begin{array}{c}\mathbf{4 4 0} \\
(0.14)\end{array}$ & $\begin{array}{c}\mathbf{2 . 5 6} \\
(0.86)\end{array}$ & - & $\begin{array}{c}1.15 \\
\text { (Fig. 6d) }\end{array}$ & $200-600$ & $2.0-3.0$ & - \\
\hline
\end{tabular}

\subsection{Time-Resolved (fluorescence) Emission Spectra (TRES)}

More details about possible relaxation paths were obtained from analysis of TRES with different delay times $\left(t_{d}\right)$. Particular importance for us is an analysis of three (lifetime) component TRES of neat AF at low temperature. In the Figure 7, TRES for neat AF at $77 \mathrm{~K}$ are presented in short (Figure 7a) and long (Figure 7b) $t_{d}$ intervals. One can see that for initial increase of $t_{d}$ the fluorescence spectra become narrower and the maximum redshifts from 17615 to $17550 \mathrm{~cm}^{-1}$ (from 568 to $570 \mathrm{~nm}$ ). The fast component quickly descents with increase of $t_{d}$, and the bands with longer (ns) lifetimes ( $\tau_{2}$ and $\left.\tau_{3}\right)$ prevail in the spectra. An increase of $t_{d}$ from 1.2 to $14.85 \mathrm{~ns}$ is accompanied with the additional redshift from 17550 to $17360 \mathrm{~cm}^{-1}$ (from 570 to 576 $\mathrm{nm}$ ) and considerable band widening (Figure 7b). The wider spectral bands are mostly resulted from increased contribution of the slow component $\left(\tau_{3}\right)$, which is connected to presence of polar medium around. The widening of bands in TRES helped to consider a donor-acceptor nature of the slow $\left(\tau_{3}\right)$ component.

Differential TRES analysis for AF at $77 \mathrm{~K}$ has allowed us to fit all the spectra in Figure 7 with superposition of three spectral components constituting: fast $\left(\tau_{1}=150-300 \mathrm{ps}\right)$, intermediate $\left(\tau_{2}=1.5-2.6 \mathrm{~ns}\right)$, and slow $\left(\tau_{3}=3.5-10 \mathrm{~ns}\right)$ components. The spectral shape of the fast $\left(\tau_{1}\right)$ and intermediate $\left(\tau_{2}\right)$ components is obtained from differential TRES (curve 6, Figure 7a and curve 7, Figure 7b), whereas the slow $\left(\tau_{3}\right)$ spectral component is practically represented by fluorescence spectra with $t_{d}$ at $10 \mathrm{~ns}$ and more (curve 6 , Figure $7 \mathrm{~b}$ ). The differential spectra do not reflect the precise spectral dependence, but allow us to estimate the fluorescence maxima $\left(\lambda_{\max }^{F L}\right)$ and Stokes shifts for different decay times. According to the Figure 7, the $\lambda_{\max }^{F L}$ of the fast component $\left(\tau_{1}\right)$ is $17750 \mathrm{~cm}^{-1}(563 \mathrm{~nm})$, the peak of intermediate component $\left(\tau_{2}\right)$ is $17600 \mathrm{~cm}^{-1}(568$ 
$\mathrm{nm})$, and the $\lambda_{\max }^{F L}$ of slow component $\left(\tau_{3}\right)$ is $17360 \mathrm{~cm}^{-1}(576 \mathrm{~nm})$. This way, we able to calculate the difference of Stokes shifts $(\Delta)$ between the components: $\Delta=150 \mathrm{~cm}^{-1}$ between $\tau_{1}$ and $\tau_{2}$ components, and $\Delta=$ $390 \mathrm{~cm}^{-1}$ between $\tau_{1}$ and $\tau_{3}$ components.

(a)

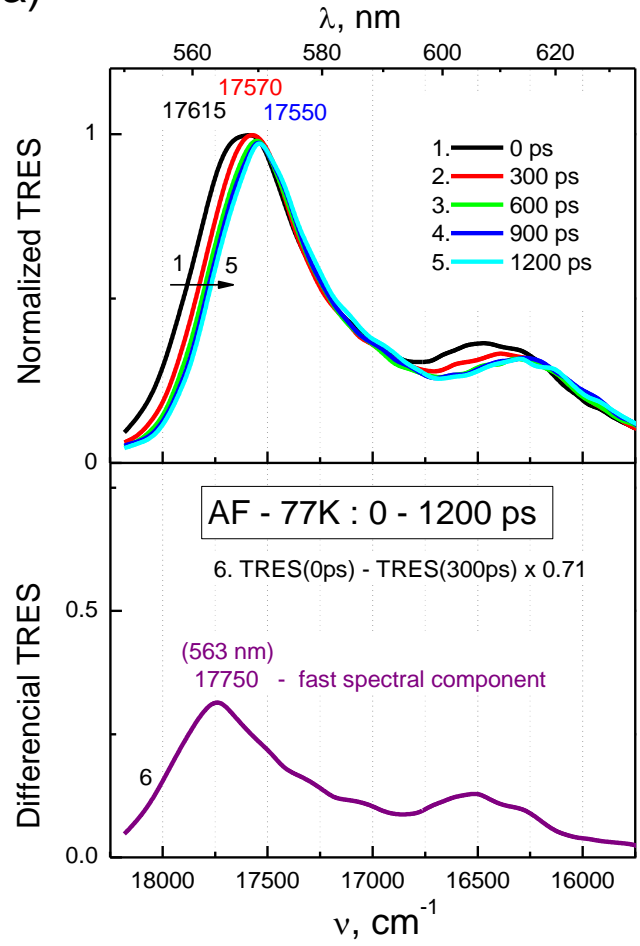

(b)

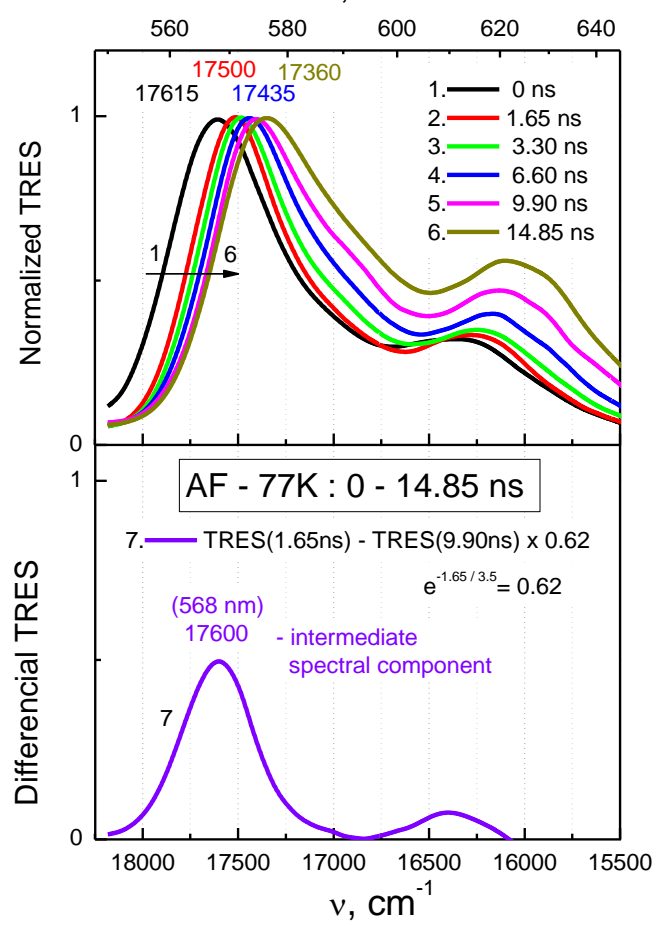

Figure 7. Normalized TRES for aqueous solution of AF at $77 \mathrm{~K}$ at $t_{d}=0-1200 \mathrm{ps}$ (a) and 0-14.85 ns (b).

The differential TRES correspond to fast $\left(\tau_{1}\right)$ and intermediate $\left(\tau_{2}\right)$ spectral components (Table 2 ).

(a) The fast component (curve 6) obtained by subtraction from the zero-delay (curve 1) of the 300 ps delayed spectrum (curve 2) normalized to a sum of $\alpha_{i}$ for $\tau_{2}$ and $\tau_{3}: 0.36+0.35=0.71$ (Table 2).

(b) The intermediate spectral component (curve 7) obtained by subtraction from the 1.65 ns delayed spectrum (curve 2) of the $9.9 \mathrm{~ns}$ delay spectrum (curve 5) normalized to a factor of $\mathrm{e}^{-1.65} / 3.5=0.62$.

Such normalization is justified by the fact that at $1.65 \mathrm{~ns}$ the fast component $\left(\tau_{1}\right)$ is negligibly small, and slow $\left(\tau_{3}=3.5 \mathrm{~ns}\right)$ component is lowered by $\mathrm{e}^{-1.65 / 3.5}=0.62$, respectively.

TRES analysis for AF with SDS is simplified due to the presence of two lifetime components and a very small shift between fast $\left(\tau_{1}\right)$ and slow $\left(\tau_{2}\right)$ spectral components. The $\lambda_{\text {max }}^{F L}$ of the fast spectral component $\left(\tau_{1}=200-600 \mathrm{ps}\right)$ is $17800 \mathrm{~cm}^{-1}(562 \mathrm{~nm})$, whereas the slow TRES spectrum $\left(\tau_{2}=2.0-3.0 \mathrm{~ns}\right)$ has peak at $17570 \mathrm{~cm}^{-1}(569 \mathrm{~nm})$. It should be noted that the peak of the slow component practically does not shift with $t_{d}$. The Stokes shifts difference is $\Delta=180 \mathrm{~cm}^{-1}$, which in addition to same time scale of $\tau_{2}=2-3 \mathrm{~ns}$ (Table 2) also confirms our assignment of slow $\left(\tau_{2}\right)$ spectral component in AF with SDS and intermediate $\left(\tau_{2}\right)$ spectral 
component in AF to the same relaxation pathway. Thus, TRES analysis for the low temperature measurements supports the outcomes of fluorescence decays fitting that showed three radiative pathways for excited state relaxation in highly polar medium of water and two pathways in the non-polar shell of SDS micelles.

\subsection{Possible relaxation paths: lifetimes and quantum-chemical model}

Analysis of lifetimes and TRES spectra for AF molecule allows us to propose a general diagrammatic scheme for relaxation paths of the excited states. The proposed energy diagram does not reflect all photonic processes in the molecule, but gives a comprehensive overview of fluorescent relaxation paths in cyanine dyes (Figure 8). After absorption of light with high enough energy, very fast sub-picosecond vibrational relaxation ${ }^{18}$ occurs to the lowest non equilibrium FC excited state with energy $E_{F C}^{+}$(the dashed curve represents $E^{+}{ }_{F C}$ influenced by frozen solvation shell at low temperature, e.g. $77 \mathrm{~K}) . E_{F C}^{+}$state characterizes ideal polymethinic structure of the molecule. In TRES spectra, fluorescence transition from $E_{F C}^{+}$has short wavelength peak position and lifetime corresponding to the fast component with $\tau_{1}$ (30-600 ps).

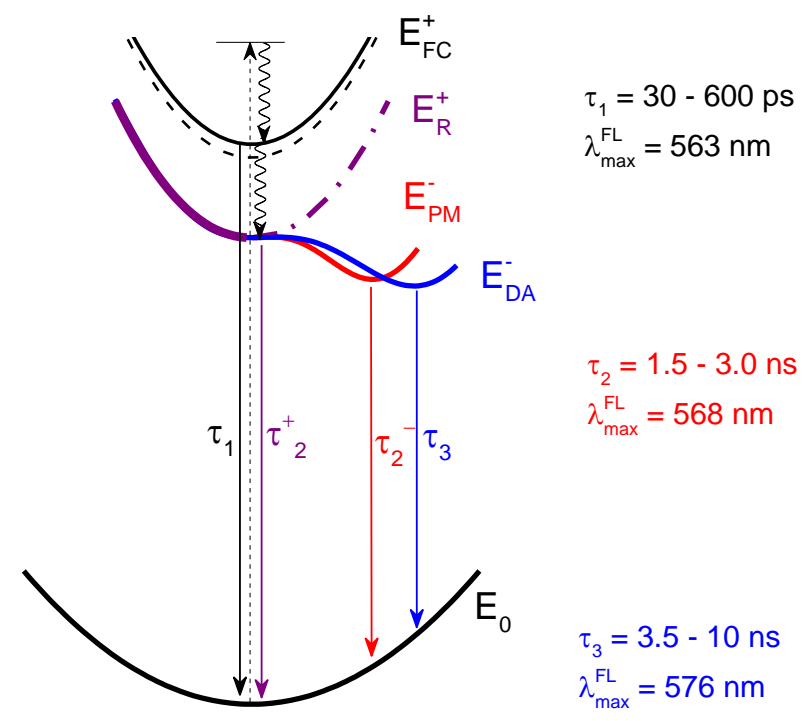

Figure 8. The energy diagram for relaxation paths of the fluorescent excited states with different $\tau_{i}$. $E_{0}$ is ground state, $E^{+}{ }_{F C}$ is non equilibrium $\mathrm{FC}$ excited state, $E_{R}^{+}$is equilibrium excited symmetric state, $E_{P M}^{-}$ and ${E_{D A}^{-}}^{-}$are excited states with broken symmetry of polymethinic and donor-acceptor polyenic nature, respectively.

Besides the fluorescence from non-equilibrium $E^{+}{ }_{F C}$ state with shortest lifetime, the excited $\mathrm{AF}$ molecule relaxes to lowest equilibrium excited $E_{R}^{+}$state with ideal polymethinic structure as well (Figure 8). Fluorescence from the $E^{+}{ }_{R}$ excited state with energy $E^{+}{ }_{R}$ is very sensitive to solvent polarity. In the non-polar 
medium like AF covered by SDS micelle shell, the relaxation from this state occurs with lifetime of $\tau^{+}{ }_{2}$ in the range of 2-3 ns. Moreover, this transition is characterized as the intermediate spectral component having maximum at ca. $17570 \mathrm{~cm}^{-1}(569 \mathrm{~nm})$, which practically does not change on delay times $\left(t_{d}\right)$ in TRES measurements. In addition, this band is characteristic for all TRES regardless medium polarity.

In the highly polar solvent like water, the symmetry breaking occurs, and hence excited AF molecule with ideal polymethinic structure relaxes to states featuring unsymmetrical charge distribution along polymethine chain $-{\overline{E_{P M}^{-}}}^{-}$and ${\overline{E^{-}}}_{D A}$ (Figure 8 ). In case of the charge localization within polymethine chain, we still have a polymethinic system of ${E_{P M}^{-}}^{-}$state, where maximum charge alternation is located within the polymethine chain. The transitions from $E_{P M}^{-}$are characterized with fluorescence lifetime $\tau_{2}^{-}$and intermediate spectral component (Figure $7 \mathrm{~b}$ ). It should be noted that the $\tau_{2}{ }_{2}$ for polar medium of water is ranged within 1.5-2.6 ns (Table 2) being a bit faster than non-polar $\tau^{+}{ }_{2}$ (2.0-3.0 ns). In TRES, the spectra depend on delay times $\left(t_{d}\right)$ showing redshift of TRES maxima with an increase of $t_{d}$. The dependence of TRES on $t_{d}$ is explained via variety of unsymmetrical charge distributions along polymethine chain. Similar unsymmetrical relaxation path was theoretically predicted earlier considering simplest terminal groups with one $\pi$-center (amino group, $\mathrm{NH}_{2}$ ) ${ }^{18}$ In AF molecule containing complex terminal groups with branched conjugated system, an additional excited state is possible, when total positive charge is located completely within one of the terminal indolenine residues. This way, AF molecule transforms from polymethine structure with symmetrical charge distribution $\left(E^{+}{ }_{R}\right)$ or nonideal polymethine structure with weakly unsymmetrical charge distribution $\left(E_{P M}^{-}\right)$into unsymmetrical molecule with localization of charge on one of the nitrogen atoms of terminal groups $\left(E_{D A}^{-}\right)$. Due to the localization of the positive charge on the nitrogen atom of cyanine molecule, a transition occurs from the polymethine to the polyene donor-acceptor molecular structure with a long fluorescent lifetime $\left(\tau_{3}\right)$ being characteristic for neat AF in water medium only. Thus, the contributions of relaxation paths with different lifetime components strongly depend on the polarity of the surrounding media.

According to the literature, symmetrical polymethine molecule upon excitation relaxes to the ground state by two different vibrational modes: (1) symmetrical path with equalized bond lengths and a symmetric charge distribution, and (2) non-symmetrical pathway. ${ }^{18-20}$ The second relaxation path is associated with the symmetry breaking, similarly to symmetry breaking in the ground state for the polymethine dyes (1) containing crucial number of vinylene groups, $i$, in the chain. For example, the unsymmetrical path is accompanied by unsymmetrical vibronical transformation along polymethine chain, so that the bond lengths alternation appears from one TG to another (so called polyenic form of donor-acceptor state). At the same time, an emergence of the lengthened bond naturally leads to a decrease of the barrier for rotational transformations, as it pictured in the Figure 9 for AF molecule. 


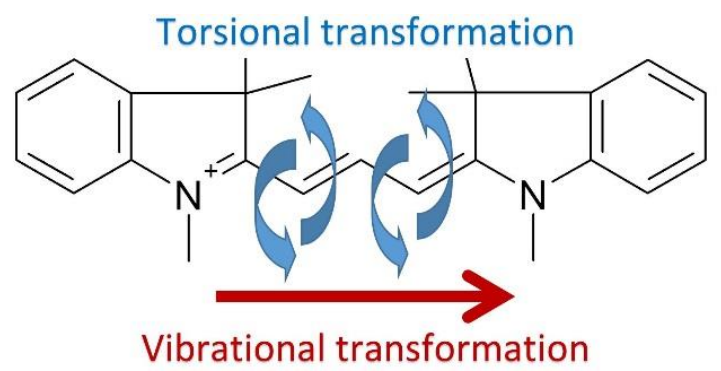

Figure 9. Possible conformational transformations in the excited state of AF.

The free molecular rotations occur in $\mathrm{AF}$ at room temperature, whereas it is impossible at low temperature and in the rigid matrix, like 5CB. Therein, we assume that only one symmetrical component of $\mathrm{AF}$ fluorescence is observed with relatively low conformational barriers at room temperature; its lifetime is in the range of 30 - 300 ps depending on the environment enclosing AF molecule. Thereafter, none unsymmetrical components should appear because of too low conformational barrier around lengthened carbon-carbon bond in the chain, thus they can rapidly transform in the radiationless forms.

At the low temperature, the conformational transformations are restricted, hence the dye molecule in the excited state remains practically planar and can efficiently emit light involving unsymmetrical pathways, i.e. manifests itself in different fluorescence spectra and lifetimes. Indeed, at the low temperature, we observed enhanced quantum yield of fluorescence, redshifted spectra and two additional slow components of fluorescence with lifetimes ( $\tau_{2}=1.5-3.0 \mathrm{~ns}$ and $\left.\tau_{3}=3.5-10 \mathrm{~ns}\right)$ in comparison with room temperature measurements (Figures 5-7, Table 2). As a consequence of detailed analysis by TRES, we have seen not two forms (as was assumed earlier ${ }^{18,19}$ ) exist in the excited state, but three distinguishable forms with the different lifetimes and spectral shapes characterize fluorescent relaxation. Thus, the comparative analysis of TRES and quantum-chemical modelling reveal that there are three components in the excited state of AF in the water solution at low temperature indicating on three radiative relaxation paths. All three forms (one symmetrical and two unsymmetrical) differ from each other by their relaxed molecular geometry and charge distribution. Briefly, the features of the electron structure of these three possible components are presented in Figure 10 and more detailed description of each path component including quantum-chemical modelling is following.

- i) Symmetrical form (Figure 10a). The total positive charge of AF is delocalized mainly on the polymethine chain; it generates the wave of the partial alternated positive and negative charges at the neighboring carbon atoms (see, for example, the Figure 3a and our previous results ${ }^{4}$ ). State dipole moments $\left(\mu^{*}\right)$ are relatively low and directed perpendicularly to the chromophore. Both TGs should be treated as donor residues because of the lone electron pair at the nitrogen atom. The bond lengths are equalized with slight alternation of its value from the chain center to TGs, so that the function of $\Delta l_{v}=(-1)^{v} \cdot\left(l_{v}-l_{v+1}\right)$, (where $v$ is number of the bond) is zero in the middle of polymethine chain (see 
the inset in Figure 10a). We propose call this form as symmetrical polymethinic state. The fast lifetime $\left(\tau_{1}=30-600 \mathrm{ps}\right)$ and short wavelength component of TRES correspond to this state.

(a)

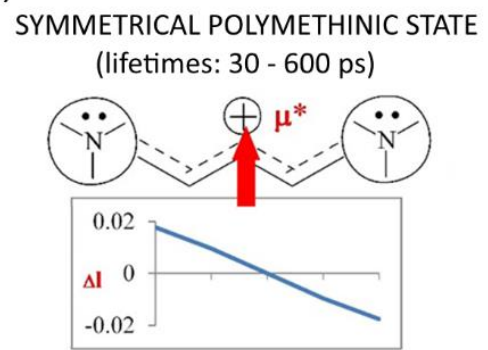

(b)

UNSYMMETRICAL POLYMETHINIC STATE

(lifetimes: 1.5 - $3.0 \mathrm{~ns}$ )
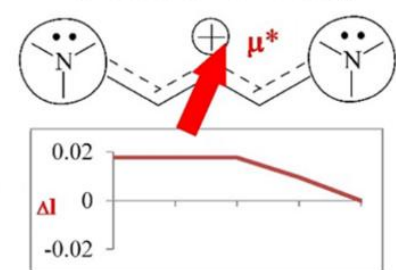

(c)

DONOR-ACCEPTOR POLYENIC STATE

(lifetimes: $3.5-10 \mathrm{~ns}$ )

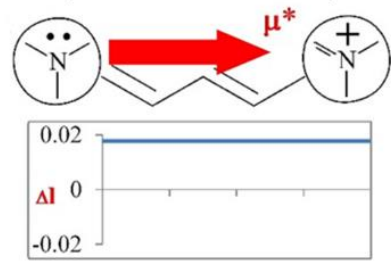

Figure 10. Electron structure of three components in the excited state of modelled polymethine chain.

- ii) Unsymmetrical form 1 (Figure 10b). The total positive charge is also delocalized mainly in the polymethine chain, but the charge distribution is unsymmetrical, so that state dipole moments $\left(\mu^{*}\right)$ are slightly inclined (for example, in Figure $10 \mathrm{~b}$ to the right side) and somewhat higher than in symmetrical form. The bond lengths remain equalized, though the function $\Delta l_{v}$ has its minimum close to one TG. We propose to call this form as unsymmetrical polymethinic state. The intermediate lifetime $\left(\tau_{2}=1.5-3.0 \mathrm{~ns}\right)$ and intermediately redshifted peaks of fluorescence relate to above form.

- iii) Unsymmetrical form 2 (Figure 10c). The positive charge is localized within one of the TGs; it is chemically presented by the formula with sign plus at the nitrogen atom. Hence, this positively charged TG should be treated as an acceptor, in contrast to another TG with the lone electron pair at 
the nitrogen atom, which should be treated as a donor. The bond length alternation, $\Delta l_{v}$, reaches its maximum with the alternation amplitude being practically regular along whole polymethine chain. Regarding to the dipole moment of theis state, its direction coincides with the chain axis (see Figure 10c). We propose to call this form as donor-acceptor polyenic state. Slow lifetime ( $\left.\tau_{3}=3.5-10 \mathrm{~ns}\right)$ and strongly redshifted peaks of TRES are characteristic features of this state.

Furthermore, we have a state with donor-acceptor properties as an analogue of merocyanines and radiation with long lifetimes of 3.5 - $10 \mathrm{~ns}$, whereas the unsymmetrical polymethinic state corresponds to intermediate lifetimes in the range of 1.5 - $3.0 \mathrm{~ns}$. We would like to underline that the donor-acceptor polyenic state is extremely sensitive to the solvent polarity. The AF molecules in 5CB or SDS micelles do not have a long lifetime component (Table 2) that can be explained via much lower polarity of local environment around AF in comparison to the highly polar water medium. In the less polar medium of 5CB or SDS micelles, nitrogen atoms do not participate in the alternation of the charges at the excitation of polymethine chain, and formation of donor-acceptor polyenic state is unlikely.

\section{Conclusions}

Thus, the performed photonic and quantum-chemical investigations show existence of three relaxation paths for symmetrical polymethine dyes in the excited state: one symmetrical path with a conservation of the symmetry for the electronic structure and two unsymmetrical paths with various symmetry breaking mechanisms. The first unsymmetrical mechanism results in the polymethinic state, whereas the second one leads to donor-acceptor polyenic state. All three components are experimentally revealed by time-resolved fluorescent spectroscopy at low temperatures in polar medium. The fluorescent paths differ by their lifetime and spectral shape (band maxima) as well as strongly depend on rigidity and polarity of environment. The studies reported here give a significantly increased fundamental understanding of molecular electronic relaxations, where high effect of matrix rigidity (viscosity) and polarity is present. There is considerable need for deep comprehension of ultrafast relaxations in such molecular systems in different environments, and we intend to continue such studies. In overall, the obtained results provide important knowledge for future design of novel photonic systems with potential application as ultrafast photonic systems, ${ }^{36}$ molecular sensors

(including fluorescence ratiometry and/or lifetime (FLIM) imaging ${ }^{3}$ ), opto-electronic switches, ${ }^{8}$ photovoltaic composites, sophisticated molecular rotors, etc.

\section{Acknowledgements}


We would like to thank cordially Prof. Yu. Slominski and Dr M. Shandura for the dye provision. P.L. acknowledges support of EU FP 'Horizon-2020' Marie Skłodowska-Curie Individual Fellowship (FOC4SIP, \#654733).

\section{References}

1. Bach G, Daehne S. Cyanine Dyes and Related Compounds. In Second Supplements to the 2nd Edition of Rodd's Chemistry of Carbon Compounds; Sainsbury, M. Ed.; Elsevier Science: Amsterdam, 1997; Vol. IV, pp 383-481.

2. Saccone, D.; Galliano, S.; Barbero, N.; Quagliotto, P.; Viscardi, G.; Barolo, C. Polymethine Dyes in Hybrid Photovoltaics: Structure-Properties Relationships, Eur. J. Org. Chem. 2016, 13, 2244-2259 and references therein.

3. Peng, X.; Yang, Zh.; Wang, J.; Fan, J.; He, Y.; Song, F.; Wang, B.; Sun, Sh.; Qu, J.; Qi, J.; Yan, M. Fluorescence Ratiometry and Fluorescence Lifetime Imaging: Using a Single Molecular Sensor for Dual Mode Imaging of Cellular Viscosity. J. Am. Chem. Soc. 2011, 133, 6626-6635.

4. Bricks, J. L.; Kachkovskii, A. D.; Slominskii, Yu. L.; Gerasov, A. O.; Popov. S. V. Molecular Design of Near Infrared Polymethine Dyes: A Review. Dyes Pigm. 2015, 121, 238-255 and references therein.

5. Karton-Lifshin, N.; Albertazzi, L.; Bendikov, M.; Baran, P. S.; Shabat, D. "Donor-Two-Acceptor” Dye Design: a Distinct Gateway to NIR Fluorescence. J. Am. Chem. Soc. 2012, 134, 20412-20420.

6. Lutsyk, P.; Arif, R.; Hruby, J.; Bukivskyi, A.; Vinijchuk, O.; Shandura, M.; Yakubovskyi, V.; Kovtun, Y.; Rance, G. A.; Fay, M., et al. A Sensing Mechanism for the Detection of Carbon Nanotubes Using Selective Photoluminescent Probes Based on Ionic Complexes with Organic Dyes. Light: Sci Appl. 2016, 4, e16028.

7. Tolbert, L. M. Solitons in a Box: the Organic Chemistry of Electrically Conducting Polyenes. Acc. Chem. Res. 1992, 25, 561-568.

8. Reimers, J. R.; Craw, J. S.; Bacskay, G. B.; Hush, N. S. Towards Efficient Molecular Wires and Switches: the Brooker Ions. Biosystems 1995, 35, 107-111.

9. Craw, J. S.; Reimers, J. R., Bacskay, G. B.; Wong, A. T.; Hush, N. S. Soliton in Finite- and InfiniteLength Negative-Defect Trans-Polyacetylene and the Corresponding Brooker (Polymethine Cyanine) Cations. I. Geometry. Chem. Phys. 1992, 167, 77-99.

10. Craw, J. S.; Reimers, J. R., Bacskay, G. B.; Wong, A. T.; Hush, N. S. Soliton in Finite- and InfiniteLength Negative-Defect Trans-Polyacetylene and the Corresponding Brooker (Polymethine Cyanine) Cations. II. Charge Density Wave. Chem. Phys. 1992, 167, 101-109. 
11. Bouit, P.-A.; Aronica, C.; Toupet, L.; Le Guennic, B.; Andraud, C.; Maury, O. Continuous Symmetry Breaking Induced by Ion Pairing Effect in Heptamethine Cyanine Dyes: Beyond the Cyanine Limit. $J$. Am. Chem. Soc. 2000, 132, 4328-4335.

12. Terenziani, F.; Przhonska, O. V.; Webster, S.; Padilha, L. A.; Slominsky, Y. L.; Davydenko, I. G.; Gerasov, A. O.; Kovtun, Y. P.; Shandura, M. P.; Kachkovski, A. D. et al. Essential-State Model for Polymethine Dyes: Symmetry Breaking and Optical Spectra. J. Phys. Chem. Lett. 2010, 1, 1800-1804.

13. Hu, H.; Przhonska, O. V.; Terenziani, F.; Painelli, A.; Fishman, D.; Ensley, T. R.; Reichert, M.; Webster, S.; Bricks, J. L. et al. Two-Photon Absorption Spectra of a Near-Infrared 2-Azaazulene Polymethine Dye: Solvation and Ground-State Symmetry Breaking. Phys.Chem. Chem. Phys., 2013, 15, 7666-7678.

14. Kachkovskii, A. D. The Solitonic Nature of Ions of Linear Conjugated Systems. Theor. Exp. Chem. 2005, 41, 139-164.

15. Ryabitski, A. B.; Kachkovski, A. D.; Przhonska O. V. Symmetry Breaking in Cationic and Anionic Polymethine Dyes. J. Mol. Struct.: THEOCHEM 2007, 802, 75-83.

16. Fabian, J. Symmetry-Lowering Distortion of Near-Infrared Polymethine Dyes - a Study by FirstPrinciples Methods. J. Mol. Struct.: THEOCHEM 2006, 766, 49-60.

17. Tolbert, L. M.; Zhao, X. Beyond the Cyanine Limit: Peierls Distortion and Symmetry Collapse in a Polymethine Dye. J. Am. Chem. Soc. 1997, 119, 3253-3258.

18. Sanchez-Galvez, A.; Hunt, P., Robb, M. A.; Olivucci, M.; Vreven, T.; S chlegel, H. B. Ultrafast Radiationless Deactivation of Organic Dyes: Evidence for a Two-State Two-Mode Pathway in Polymethine Cyanines. J. Am. Chem. Soc. 2000, 122, 2911-2924.

19. Vasylyuk, S. V.; Yashchuk, V. M.; Viniychuk, O. O.; Piryatinski, Yu. P.; Sevryukova M. M.; Gerasov, A. O.; Zyabrev, K. V.; Kovtun Yu. P.; Shandura, M. P.; Kachkovsky, O. D. The Investigation of Relaxation Paths in Dioxaborine Anionic Polymethine Dyes Detected by Low-Temperature TimeResolved Fluorescence. Mol. Cryst. Liq. Cryst. 2011, 535, 123-131.

20. Gieseking, R. L.; Ravva, M. K.; Coropceanu, V.; Brédas, J.-L. Benchmarking Density Functional Theory Approaches for the Description of Symmetry Breaking in Long Polymethine Dyes. J. Phys. Chem. C 2016, 120, 9975-9984.

21. Boom, K.; Müller, M.; Stein, F.; Ernst, St.; Morgenstern, K. Adsorption of a Switchable Industrial Dye on $\mathrm{Au}(111)$ and $\operatorname{Ag}(111)$. J. Phys. Chem. C 2015, 119, 17718-17724.

22. Mukerjee, P.; Mysels, K. J. Critical Micelle Concentrations of Aqueous Surfactant Systems. U.S. National Bureau of Standards: Washington, D.C., 1971.

23. Aniansson, E. A. G.; Wall, S. N.; Almgren, M.; Hoffmann, H.; Kielmann, I.; Ulbricht, W.; Zana, R.; Lang, J.; Tondre C. Theory of the Kinetics of Micellar Equilibria and Quantitative Interpretation of 
Chemical Relaxation Studies of Micellar Solutions of Ionic Surfactants. J. Phys. Chem. 1976, 80, 905922.

24. Humphry-Baker, R.; Grätzel, M.; Steiger, R. Drastic Fluorescence Enhancement and Photochemical Stabilization of Cyanine Dyes through Micellar Systems. J. Am. Chem. Soc. 1980, 102, 847-848.

25. Lutsyk, P.; Piryatinski, Yu.; AlAraimi, M.; Arif, R.; Shandura, M.; Kachkovsky, O.; Verbitsky, A.; Rozhin. A. Emergence of Additional Visible Range Photoluminescence Due to Aggregation of Cyanine Dye - Astraphloxin on Carbon Nanotubes Dispersed with Anionic Surfactant. J. Phys. Chem. C 2016, 120, 20378-20386.

26. Frisch, M. J.; Trucks, G. W.; Schlegel, H. B.; Scuseria, G. E.; Robb, M. A.; Cheeseman, J. R.; Montgomery, J. A., Jr.; Vreven, T.; Kudin, K. N.; Burant, J. C. et al. Gaussian 03, Revision B.05; Gaussian, Inc.: Wallingford, CT, 2003.

27. Fabian J. TDDFT-Calculations of Vis/NIR Absorbing Compounds. Dyes Pigm. 2010, 84, 36-53.

28. Karaca, S; Elmaci N. A Computational Study on the Excited State Properties of a Cationic Cyanine Dye: TTBC. Comput. Theor. Chem. 2011, 964, 160-168.

29. Jacquemin, D.; Zhao, Ya.; Valero, R.; Adamo, C.; Ciofini, I.; Truhlar D. G. Verdict: Time-Dependent Density Functional Theory "Not Guilty" of Large Errors for Cyanines. J. Chem. Theory Comput. 2012, $8,1255-1259$.

30. Lackowicz, J. R. Principles of Fluorescence Spectroscopy; 3rd edition, Springer: US, 2006.

31. Kachkovskii, A. D. The Nature of Electronic Transitions in Linear Conjugated Systems. Russ. Chem. Rev. 1997, 66, 647-664.

32. Ecker, H. Das Absorptionsspektrum von Reversiblen Polymerisaten aus Chinolinfarbstoffen. KolloidZeitschrift 1940, 92, 35-70. (In German).

33. Silva, G. L.; Ediz, V.; Yaron, D.; Armitage, B. A. Experimental and Computational Investigation of Unsymmetrical Cyanine Dyes: Understanding Torsionally Responsive Fluorogenic Dyes. J. Am. Chem. Soc. 2007, 129, 5710-5718.

34. Bogi, A.; Faetti, S. Elastic, Dielectric and Optical Constants of 4'-Pentyl-4-Cyanobiphenyl. Liq. Cryst. 2001, 28, 729-739.

35. Berezin, M. Y.; Lee, H.; Akers, W.; Achilefu, S. Near Infrared Dyes as Lifetime Solvatochromic Probes for Micropolarity Measurements of Biological Systems. Biophys. J. 2007, 93, 2892-2899.

36. Piatkowski, L.; Accanto, N.; van Hulst, N. F. Ultrafast Meets Ultrasmall: Controlling Nanoantennas and Molecules. ACS Photonics 2016, 3, pp 1401-1414. 
TOC Graphic

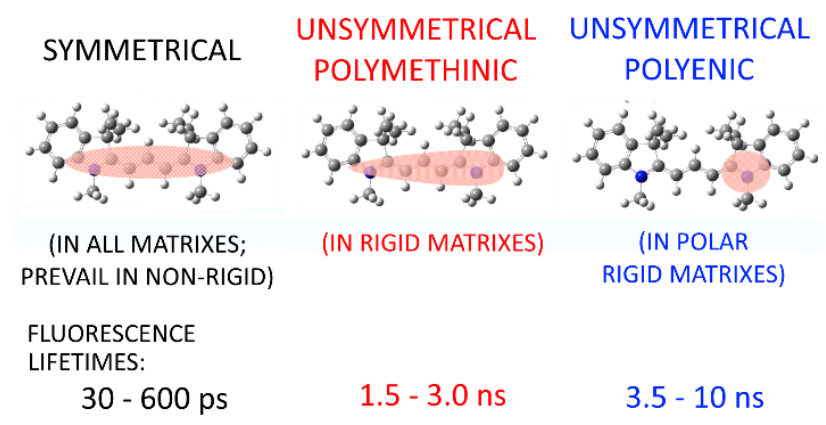

